patient's journey and concern that the care given is appropriate. It also appears that most of the factors that help are the mirror images of those that hinder the process of deciding when the pathway should be implemented. The theme remains the same but the meaning as to whether it helps the clinician to decide if a patient is dying or not changes.

Conclusions All clinicians interviewed for this study had concerns about increasing the patient's or carers' distress if LCP implementation was mistimed; there is a risk that clinicians are avoiding difficult conversations with family members and there may be a lack of understanding around the reasons for it's use. Specific communications training may help to prepare clinicians for this role.

\section{P136 \\ DOING THE RIGHT THING AT THE RIGHT TIME}

Joan Dee St Luke's Hospice, Plymouth, Devon

10.1136/bmjspcare-2011-000105.136

Introduction In order to improve the care of patients and their families when the patient is dying it is helpful to implement the Liverpool Care Pathway for the Dying. It therefore is necessary to identified them in a timely fashion. Previous studies suggest that physicians tend to be overly optimistic when attempting to predict survival.

Objective To identify factors that clinicians consider when a patient is dying,

enabling implementation of the Liverpool Care Pathway.

Method A phenomenological study using semi-structured interviews ( $\mathrm{n}=$ five nurses and five doctors) conducted on a hospice inpatient unit.

Results There was a prominent theme of anxiety about getting the timing of diagnosing dying right and how the dying patient and their families would cope if this were the case. The factors that influenced the clinician's decision to implement the LCP were: support for decision making, understanding the 